\title{
An Iterative MIMO-HSDPA Receiver Based On a K-Best-MAP Algorithm
}

\author{
Christian Mehlführer, Dominik Seethaler, Gerald Matz, and Markus Rupp \\ Institute of Communications and Radio-Frequency Engineering \\ Vienna University of Technology \\ Gusshausstrasse 25/389, A-1040 Vienna, Austria \\ Email: \{chmehl, dseethal, gmatz,mrupp\}@nt.tuwien.ac.at \\ Web: http://www.nt.tuwien.ac.at
}

\begin{abstract}
We propose an iterative receiver for horizontally encoded MIMO systems like the PARC proposal for MIMOHSDPA. Our receiver uses a K-best-MAP demodulator to provide the channel decoder with approximate log-likelihood ratios. The complexity of K-best-MAP is reduced by exploiting the CRC present in each encoded layer. We show that K-best-MAP with list update is superior to demodulation using a fixed candidate list. Finally, our numerical results demonstrate significant SNR gains as compared to a receiver using MMSE-based successive interference cancelation.
\end{abstract}

\section{INTRODUCTION}

In this paper, we propose an efficient iterative MIMO receiver based on a K-best-MAP algorithm. Our scheme is applicable to any horizontally encoded MIMO system where all layers are encoded individually. However, the focus will be on the per-antenna-rate-control (PARC) proposal [1] for the MIMO extension of HSDPA (High Speed Downlink Packet Access) [2]. Our approach is motivated by excellent performance results of iterative receivers in MIMO wireless systems [3]. The iterative receiver consists of a MIMO demodulator and a channel decoder which exchange extrinsic loglikelihood ratios (LLRs). Since optimum MIMO demodulation has exponential complexity, various efficient approximations have been proposed. Among those, promising algorithms are based on sphere decoding [3]-[6] and systematic tree search [7], [8].

In [3], [4], the list sphere decoder (LSD) is used to generate a candidate list of transmitted symbol vectors. The list is then used to calculate LLRs of the coded bits. Although the LSD performs very well, it features two drawbacks. First, the complexity of the LSD is not fixed but rather depends strongly on the channel realization and the SNR [9]. This is a particular problem if it is not feasible to average complexity during runtime, e.g., if buffer size is small or if the channel changes slowly compared to the frame duration. The second problem of the LSD is the choice of the initial search radius. When the radius is small, too few candidate vectors may be found; when the radius is too large, many distance metrics have to be evaluated, thus increasing complexity.

Both disadvantages of the LSD can be avoided by using the K-best algorithm introduced in [10] to generate a list of candidate vectors. The K-best algorithm furthermore is very attractive for parallel implementations since it performs just a tree search in forward direction. In Section II, we thus propose an iterative MIMO-HSDPA receiver that uses a K-best-MAP algorithm (cf. [5], [6]) for demodulation. We exploit the horizontal encoding structure by using the cyclic redundancy check (CRC) bits within each layer to further decrease the computational complexity of the K-best-MAP demodulator. Two versions of K-best-MAP are investigated within our iterative receiver. One performs an update of the candidate list based on a priori LLRs and the other uses the same candidate list for all iterations. To achieve a near optimum performance, the receiver without list update will be seen to require a much larger list size but less receiver iterations than the one doing a list update. A complexity assessment reveals that indeed the receiver with list update has smaller computational complexity.

In Section IV, we present numerical results for the PARC MIMO-HSDPA proposal [1]. Different from the vertically encoded MIMO systems in [3]-[8], PARC uses horizontal encoding. This has the advantage of allowing for separate retransmission of erroneous layers. The performance of our receiver is evaluated in terms of system throughput. We observe that at the target throughput our approach suffers virtually no loss as compared to the optimal iterative receiver and yields a $3 \mathrm{~dB}$ SNR gain over an MMSE successive interference cancelation (MMSE-SIC) receiver (as proposed in [2]).

\section{A. System model}

We consider a coded MIMO system with $N_{T}$ transmit antennas and $N_{R} \geq N_{T}$ receive antennas as in the PARC proposal for the MIMO extension of UMTS [2]. Here, each data stream on each transmit antenna (or layer) is individually encoded using a rate $1 / 3$ turbo code followed by an interleaver. Additional CRC bits are added to each layer to detect packet errors. Finally, Gray labeling is used to map the coded and interleaved bits in the $k$ th layer to complex data symbols $s_{k} \in \mathcal{A}$ ( $\mathcal{A}$ denotes the symbol alphabet).

A flat-fading MIMO channel is assumed. At any symbol time instant, the baseband model is given by

$$
\mathbf{x}=\mathbf{H} \mathbf{s}+\mathbf{n},
$$




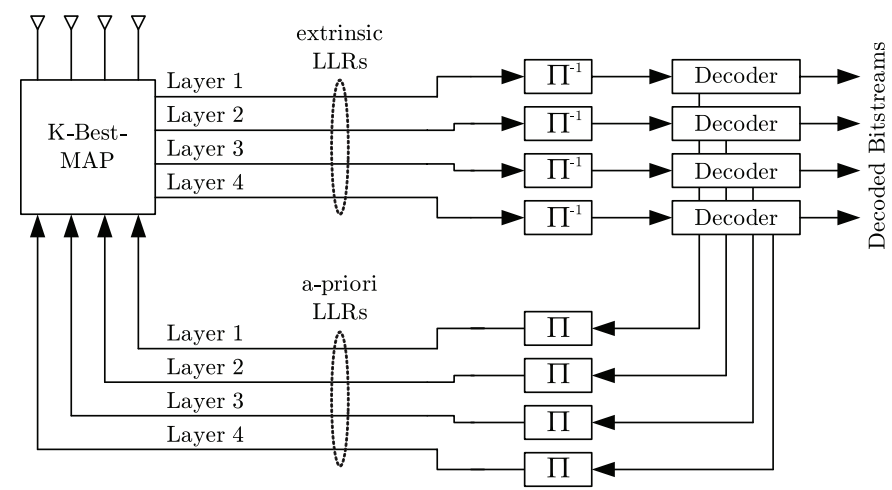

Fig. 1. Iterative MIMO-HSDPA receiver using the K-best-MAP algorithm for a $4 \times 4$ horizontally encoded MIMO system with four data streams.

with the transmitted data symbol vector $\mathbf{s}=\left(s_{1} \cdots s_{N_{T}}\right)^{T}$, the $N_{R} \times N_{T}$ channel matrix $\mathbf{H}$, the received vector $\mathbf{x}=$ $\left(x_{1} \cdots x_{N_{R}}\right)^{T}$, and the noise vector $\mathbf{n}=\left(n_{1} \cdots n_{N_{R}}\right)^{T}$. The noise components $n_{k}$ are assumed independent and circularly symmetric complex Gaussian with variance $\sigma_{n}^{2}$. The flat fading scenario is representative for indoor MIMO-HSDPA transmissions. With an OFDM physical layer, currently evaluated by the 3GPP for future UMTS releases, the same model applies to frequency selective channels.

\section{Proposed Receiver}

The proposed iterative MIMO receiver is shown in Fig. 1. Here, a K-best-MAP demodulator (see below) calculates LLRs for the coded bits of each layer. The extrinsic part of these LLRs are deinterleaved and serve as bit metrics for the soft-input soft-output turbo decoders within each layer. The extrinsic output LLRs of the turbo decoders are re-interleaved and used as a priori LLRs for the K-best-MAP demodulator. The soft demodulation and soft decoding process is repeated for $N_{\text {rec }}$ receiver iterations. Within each receiver iteration, the turbo decoders themselves perform $N_{\text {turbo }}$ iterations.

\section{A. Demodulation}

In the following, we develop a K-best-MAP demodulator that calculates LLRs for a given time instant based on (1) and on the a priori LLRs provided by the turbo decoders.

1) Max-log-MAP Demodulation: Let $b_{k, i} \in\{-1,+1\}$, $i=1, \ldots, M$, with $M=\log _{2}|\mathcal{A}|$ denote the coded bits associated to the symbol $s_{k}$ in the $k$ th layer. Using the max$\log$ approximation, the LLR of $b_{k, i}$ can be written as (e.g. [3])

$$
\Lambda_{k, i}=\min _{\mathbf{s} \in \mathcal{S}_{k, i}^{(-1)}} \psi^{2}\left(\mathbf{s}, \boldsymbol{\Lambda}^{\mathrm{A}}\right)-\min _{\mathbf{s} \in \mathcal{S}_{k, i}^{(+1)}} \psi^{2}\left(\mathbf{s}, \boldsymbol{\Lambda}^{\mathrm{A}}\right),
$$

with

$$
\psi^{2}\left(\mathbf{s}, \boldsymbol{\Lambda}^{\mathrm{A}}\right)=\frac{1}{\sigma_{n}^{2}}\|\mathbf{x}-\mathbf{H} \mathbf{s}\|^{2}-\frac{1}{2} \sum_{k=1}^{N_{T}} \sum_{i=1}^{M} b_{i}\left(s_{k}\right) \Lambda_{k, i}^{\mathrm{A}} .
$$

Here, $\mathcal{S}_{k, i}^{(b)}$ denotes the set of all transmit vectors s such that the bit label of the symbol $s_{k}$ in the $k$ th layer at bit position $i$ (denoted $b_{i}\left(s_{k}\right)$ ) equals $b$. Furthermore, $\boldsymbol{\Lambda}^{\mathrm{A}}$ refers to the $N_{T} \times M$ matrix containing the a priori LLRs $\Lambda_{k, i}^{\mathrm{A}}$ provided by the turbo decoders (during the first pass, $\Lambda_{k, i}^{\mathrm{A}}=0$ ).
2) K-best-MAP Demodulation: Since the computational complexity of (2) is exponentially in $N_{T}$, we use a K-bestMAP demodulator (similar to [5], [6]). The K-best-MAP constructs a reduced search set $\mathcal{L} \subset \mathcal{A}^{N_{T}}$ with $|\mathcal{L}| \ll|\mathcal{A}|^{N_{T}}$ that contains data vectors $\mathbf{s}$ that are good candidates with respect to achieving small values of $\psi^{2}\left(\mathbf{s}, \boldsymbol{\Lambda}^{\mathrm{A}}\right)$ in (3). The LLRs can then be approximated by

$$
\Lambda_{k, i} \approx \min _{\mathbf{s} \in \mathcal{S}_{k, i}^{(-1)} \cap \mathcal{L}} \psi^{2}\left(\mathbf{s}, \Lambda^{\mathrm{A}}\right)-\min _{\mathbf{s} \in \mathcal{S}_{k, i}^{(+1)} \cap \mathcal{L}} \psi^{2}\left(\mathbf{s}, \boldsymbol{\Lambda}^{\mathrm{A}}\right) .
$$

The size of the reduced search set $\mathcal{L}$ (and thus the computational complexity of the algorithm) is given by $|\mathcal{L}|=K$ (optimum max-log-MAP demodulation is equivalent to $\mathrm{K}$ best-MAP with $K=|\mathcal{A}|^{N_{T}}$ ). Its construction is achieved by incorporating the a priori LLRs into K-best sphere decoding [10] as follows.

We use the QR-factorization of the channel matrix

$$
\mathbf{H}=\mathbf{Q}\left[\begin{array}{c}
\mathbf{R} \\
\mathbf{0}
\end{array}\right],
$$

where $\mathbf{Q}$ and $\mathbf{R}$ refer to an $N_{R} \times N_{R}$ unitary matrix and an $N_{T} \times N_{T}$ upper triangular matrix, respectively. By defining the $N_{R} \times N_{T}$ matrix $\mathbf{Q}_{1}$ and the $\left(N_{R}-N_{T}\right) \times N_{T}$ matrix $\mathbf{Q}_{2}$ via $\mathbf{Q}=\left[\mathbf{Q}_{1} \mathbf{Q}_{2}\right]$, we can write (3) as

$$
\begin{aligned}
& \psi^{2}\left(\mathbf{s}, \boldsymbol{\Lambda}^{\mathrm{A}}\right)= \\
& \sum_{k=1}^{N_{T}}\left(\frac{1}{\sigma_{n}^{2}}\left|(\mathbf{y})_{k}-(\mathbf{R s})_{k}\right|^{2}-\frac{1}{2} \sum_{i=1}^{M} b_{i}\left(s_{k}\right) \Lambda_{k, i}^{\mathrm{A}}\right)+C,
\end{aligned}
$$

where $\mathbf{y}=\mathbf{Q}_{1} \mathbf{x}$ and $(.)_{k}$ denotes he $k$ th element of a vector. Since $C=\left\|\mathbf{Q}_{2}^{H} \mathbf{x}\right\|^{2}$ does not affect the minimizations in (4), it will be ignored in the following.

The triangular matrix $\mathbf{R}$ in (5) induces a search tree (see Fig. 2) that can be processed from top (layer $N_{T}$ ) to bottom (layer 1) in order to recursively determine small values of $\psi^{2}\left(\mathbf{s}, \boldsymbol{\Lambda}^{\mathrm{A}}\right)$ with associated candidate vectors, similar to classical K-best sphere decoding. The main difference is that the partial metrics are augmented by the a priori LLRs. For example, the partial metrics for $k=N_{T}$ (top of tree) and $s_{N_{T}} \in \mathcal{A}$ equal

$$
\frac{1}{\sigma_{n}^{2}}\left|y_{N_{T}}-R_{N_{T}, N_{T}} s_{N_{T}}\right|^{2}-\frac{1}{2} \sum_{i=1}^{M} b_{i}\left(s_{N_{T}}\right) \Lambda_{N_{T}, i}^{\mathrm{A}},
$$

which involves just the $N_{T}$-th layer. We can immediately select the $K$ data symbols $s_{N_{T}}$ that achieve the minimum partial metric. This process can then be repeated recursively by proceeding to the next layer whose partial metric depends only on the symbols in the current layer and the fixed symbols selected in the previous layers. In that way, $K$ data vectors constituting the reduced search list $\mathcal{L}$ are determined when the first layer (bottom of tree) is reached. At each layer (tree level) $\min \left\{|\mathcal{A}|^{k}, K|\mathcal{A}|\right\}$ partial metrics are evaluated and $K$ symbols are selected. Note that the data vectors in $\mathcal{L}$ are in general not the overall $K$ best data vectors in the sense that they achieve the $K$ smallest values of $\psi^{2}\left(\mathbf{s}, \boldsymbol{\Lambda}^{\mathrm{A}}\right)$ in (3). Nonetheless, excellent performance results can be achieved even for small values of $K$ (see Section IV). 


\section{B. Modifications and Extensions}

1) Incorporating the $C R C$ : In addition to the turbo code, each layer is protected by a CRC to detect packet errors. In the case of a packet error, only the erroneous layer has to be retransmitted which is an advantage over systems using vertical encoding. The CRC is checked after each turbo decoder pass. One can think of various ways to exploit the $\mathrm{CRC}$ to improve the performance and to reduce the complexity of our iterative receiver. A straightforward approach is to perform interference cancelation of a layer whose CRC is already correct. This reduces the number of remaining active layers but results in a modified system model with a different search tree (requiring a new QR-decomposition). Thus, we propose to directly incorporate a correct CRC in the K-bestMAP with the full search tree without changing the system model. In particular, if the CRC of a certain layer is correct, we know all symbols in that layer and thus the transitions in the associated layer of the search tree. We thus skip the minimization of the partial metrics at this layer and pick the known correct symbols and associated partial metrics, thereby evaluating only $K$ instead of $K|\mathcal{A}|$ partial metrics,

2) Fixed Candidate List: Obviously, a possible approach to reduce the complexity of our iterative receiver is to use the K-best-MAP algorithm only once at the beginning of the iterative process to calculate the candidate list $\mathcal{L}$ (e.g. this is done in [3] using the LSD). This candidate list is then used within each receiver iteration to calculate the LLRs according to (4). However, we will see in Section (IV) that a fixed list necessarily has to be very large to achieve high target throughputs (however, fewer receiver iterations are necessary with a large, fixed list). A comparison of the complexity of receivers using a fixed list and list updates will be provided in Section III.

3) LLR Clipping: Sometimes it happens (in particular for small $K$ ) that $\mathcal{S}_{k, i}^{(+1)} \cap \mathcal{L}=\emptyset$ or $\mathcal{S}_{k, i}^{(-1)} \cap \mathcal{L}=\emptyset$ for some $k$ and $i$, i.e., there is no candidate vector such that $b_{i}\left(s_{k}\right)=1$ or $b_{i}\left(s_{k}\right)=-1$, respectively. In that case, one of the two minimizations in (4) cannot be performed and one has to clip the corresponding LLR to a certain value $\pm \Lambda_{\text {clip }}$ ([3] and [8] suggest the clipping levels $\Lambda_{\text {clip }}=8$ and $\Lambda_{\text {clip }}=3$, respectively). The LLR clipping level has a significant impact on performance. We circumvent the drawbacks of fixed LLR clipping by using a clipping level equal to the average of all (non-clipped) LLR magnitudes within the associated data stream. This is a robust choice useful at all SNR levels.

\section{Example for K-best-MAP}

An example for the K-best-MAP algorithm with $K=2$ and $N_{T}=4$ QPSK data streams is shown in Fig. 2 (i.e., $|\mathcal{A}|^{N_{T}}=256$ ). Only those branches are shown for which partial metrics are actually evaluated. In this example, the CRC has indicated that the second layer has already been decoded correctly leading to the symbol $s_{2}=-1$. Furthermore, both candidate vectors have $s_{3}=j$ which implies that the LLRs of the associated two bits will be subjected to clipping.

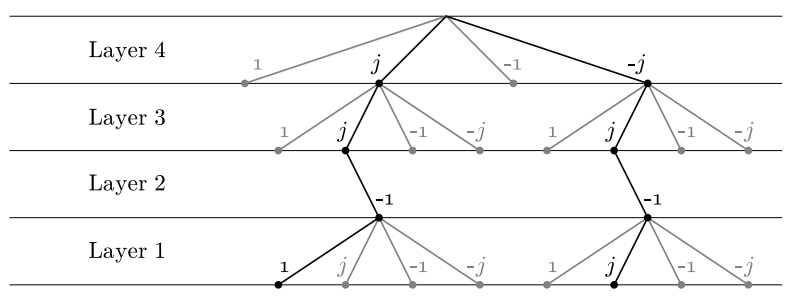

Fig. 2. Example for the K-best-MAP algorithm in a $4 \times 4$ QPSK system with candidate list size $K=2$ (not all tree branches shown). Here, $\mathcal{L}$ consists of the two candidate vectors $[1,-1, j, j]^{T}$ and $[j,-1, j,-j]^{T}$.

\section{COMPutational COMPlexity}

We next provide estimates for the computational complexity of the two main components of our receiver (namely the Kbest-MAP algorithm and the turbo decoder).

With regard to the turbo decoding process, the computational effort per information bit for max-log-MAP decoding is proportional to the number $S$ of trellis states. On the basis of 8 (real valued) flops per information bit (e.g. [11]) and rate $1 / 2$ codes we have about $C_{\text {conv }}=4 S$ flops per code bit for max-log-MAP decoding. In HSDPA, the rate $1 / 3$ turbo code [12] consists of two rate $1 / 2$ convolutional component codes. Thus, we obtain a computational effort of the turbo decoder of about $C_{\text {turbo }}=16 / 3 S$ flops per coded bit and iteration. Thus, the computational effort of the eight state HSDPA turbo decoder is approximately $C_{\text {turbo }}=43$ flops per coded bit and iteration.

For the K-best-MAP algorithm, the main computational effort is in evaluating (5). At each layer, we have up to (due to decisions confirmed by the CRC) $\min \left\{|\mathcal{A}|^{k-1}, K\right\}$ surviving nodes. An estimate of the computations involved for the processing of the $|\mathcal{A}|$ metrics of one of the surviving nodes is $8(|\mathcal{A}|+k-1)$ real valued flops at layer $k$. This yields an overall computational effort of

$$
C_{\mathrm{K}-\text { best }}=\frac{8}{N_{T} M} \sum_{k=1}^{N_{T}} \min \left\{|\mathcal{A}|^{k-1}, K\right\}(|\mathcal{A}|+k-1)
$$

flops per coded bit. This has a $\mathcal{O}\left(K N_{T}\right)$ complexity. For a $4 \times 4$ QPSK system and $K=8$ we obtain from (6) $C_{\mathrm{K} \text {-best }}=$ 128 flops per coded bit.

We conclude that K-best-MAP complexity is approximately three times larger than the complexity of one (inner) turbo decoder iteration. Thus, it should be a design goal to minimize the number $N_{\text {rec }}$ of outer receiver iterations. To achieve the same throughput, we might want to perform more turbo decoder iterations and less receiver iterations to achieve a lower computational effort for the whole receiver. We note however, that for other systems (e.g. with 64 state convolutional codes) the computational effort of the decoder might be higher than that of the K-best-MAP algorithm. Overall complexity in such a system could be optimized by increasing the number of (outer) receiver iterations and reducing the number of (inner) decoder iterations. For optimizing iterative receiver structures it is thus of utmost importance to have accurate estimates of the computational efforts of the subsystems. 
TABLE I

HSDPA SIMULATION PARAMETERS.

\begin{tabular}{|r|c|}
\hline Parameter & Value \\
\hline \hline$\hat{I}_{o r} / I_{o c}{ }^{1}$ & $10 \mathrm{~dB}$ \\
\hline Channel estimation & least squares \\
\hline turbo decoding & max-log-MAP $-N_{\text {turbo }}$ iterations \\
\hline Modulation & QPSK \\
\hline Transport block size & $4 \times 1256$ bits \\
\hline No. of channelization codes & 4 \\
\hline Maximum data rate & $2.512 \mathrm{Mbps}$ \\
\hline
\end{tabular}

\section{Simulation Results}

Our simulations were performed for an iid channel and different $E_{c} / I_{o r}$ (chip energy of the data stream over total energy radiated by the base station). The simulation parameters are summarized in Table I. For every $E_{c} / I_{o r}, 200$ HSPDA frames (1000 subframes) were transmitted [13]. The system throughput is determined by counting the number of bits in "error free" subframes (with correct CRC) and discarding the bits in erroneous subframes. Since the HSDPA specification [14] states that the user equipment shall achieve a block error rate of $10 \%$ by means of the channel quality indicator feedback, we compare the throughput at $90 \%$ of the maximum throughput for a specific channel quality value. Note that at this specific channel quality value (i.e. modulation scheme and channel coding rate) a maximum data rate of $2.512 \mathrm{Mbps}$ can be achieved when four out of 16 possible spreading codes are used. In all simulations the channel is estimated using a least squares channel estimator based on the received pilot symbols.

\section{A. Performance of the K-best-MAP Algorithm}

In the following we assume $N_{\text {turbo }}=8$ turbo decoder iterations within each receiver iteration (after eight turbo iterations the performance of the turbo decoder does no longer improve). Fig. 3 depicts the throughput performance of the iterative receiver compared to the MMSE-SIC receiver. At $90 \%$ of the maximum throughput, we observe about $3 \mathrm{~dB}$ gain in $E_{c} / I_{o r}$. Even $K=8$ and no receiver iteration yields a better performance than the MMSE-SIC receiver. Furthermore, the iterative receiver using the K-best-MAP demodulator with $K=8$ achieves a performance that is very close to the iterative receiver with full list size $(K=256)$.

The impact of list size $K$ and number of receiver iterations $N_{\text {rec }}$ is illustrated in Fig. 4. The results were obtained for a fixed $E_{c} / I_{o r}$ value of $-11 \mathrm{~dB}$, ensuring a comparison at approximately $90 \%$ of the maximum throughput. From Fig. 4 we can conclude that $K=8$ and $N_{\text {rec }}=2$ are sufficient to achieve a performance similar to the full list size demodulator. Increasing $K$ and/or $N_{\text {rec }}$ beyond these values gives only marginal performance improvement. It is also seen that the same receiver performance can be achieved e.g. by choosing $K=4$ and $N_{\text {rec }}=4$ or $K=8$ and $N_{\text {rec }}=1$.

\footnotetext{
${ }^{1}$ The ratio $\hat{I}_{o r} / I_{o c}$ relates the amount of energy received from the desired basestation to the energy received from neighboring basestations (interferers).
}

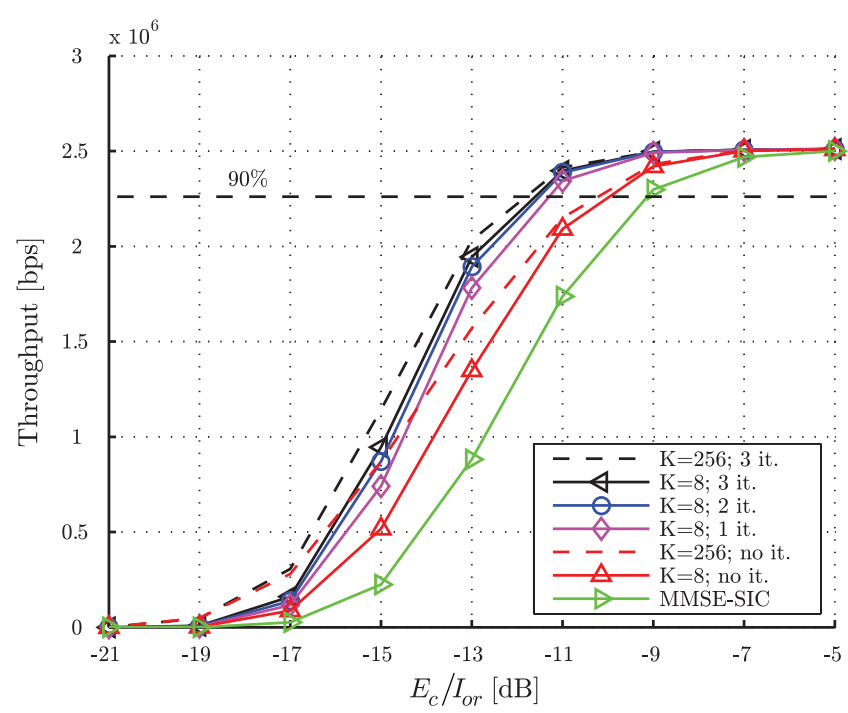

Fig. 3. Throughput versus $E_{c} / I_{\text {or }}$ for a $4 \times 4$ MIMO system with QPSK.

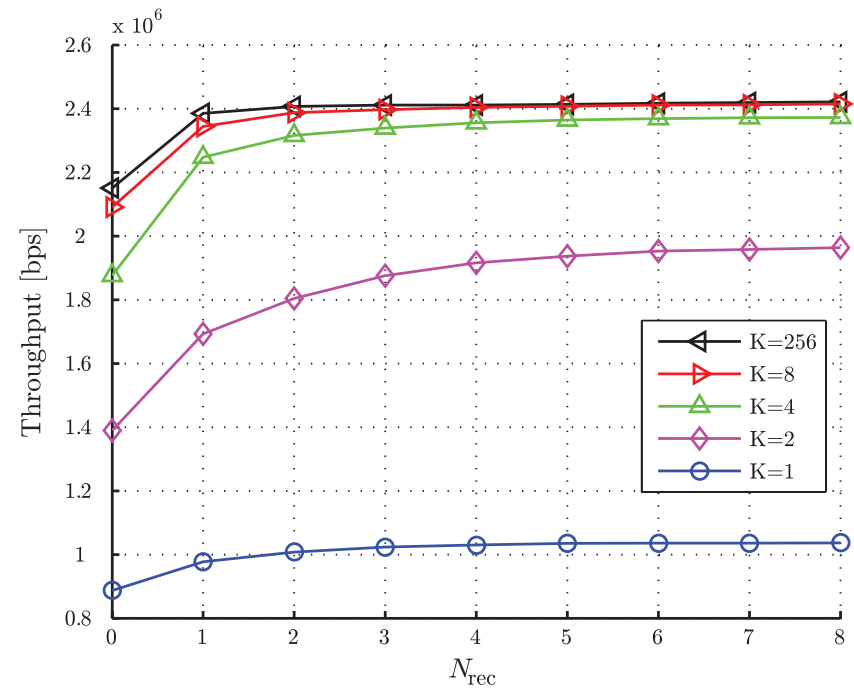

Fig. 4. Throughput versus $N_{\text {rec }}$ for various $K$ and $E_{c} / I_{o r}=-11 \mathrm{~dB}$.

\section{B. Tradeoff $N_{\text {rec }}$ versus $N_{\text {turbo }}$}

The throughput for various fixed numbers of turbo decoder iterations $N_{\text {turbo }}$ is depicted in Fig. 5. Here, every marker corresponds to an additional (outer) receiver iteration. For $N_{\text {turbo }}$ small (1 or 2), the performance increases very fast but saturates at smaller throughput values than for high values of $N_{\text {turbo }}$. According to Fig. 5 a good performance/complexity tradeoff is achieved with $N_{\text {turbo }}=8$ since this requires the fewest number of receiver iterations. The performance of $N_{\text {rec }}=2$ and $N_{\text {turbo }}=8$ can also be achieved with $N_{\text {rec }}=5$ and $N_{\text {turbo }}=2$, which however is slightly more complex. We emphasize that this result is specific to the HSDPA turbo code and a $4 \times 4$ QPSK transmission with $K=8$ K-best-MAP detection (cf. the discussion in Section III).

\section{Fixed Candidate List}

The throughput performance of an iterative receiver with fixed candidate list is shown in Fig. 6. It can be observed 


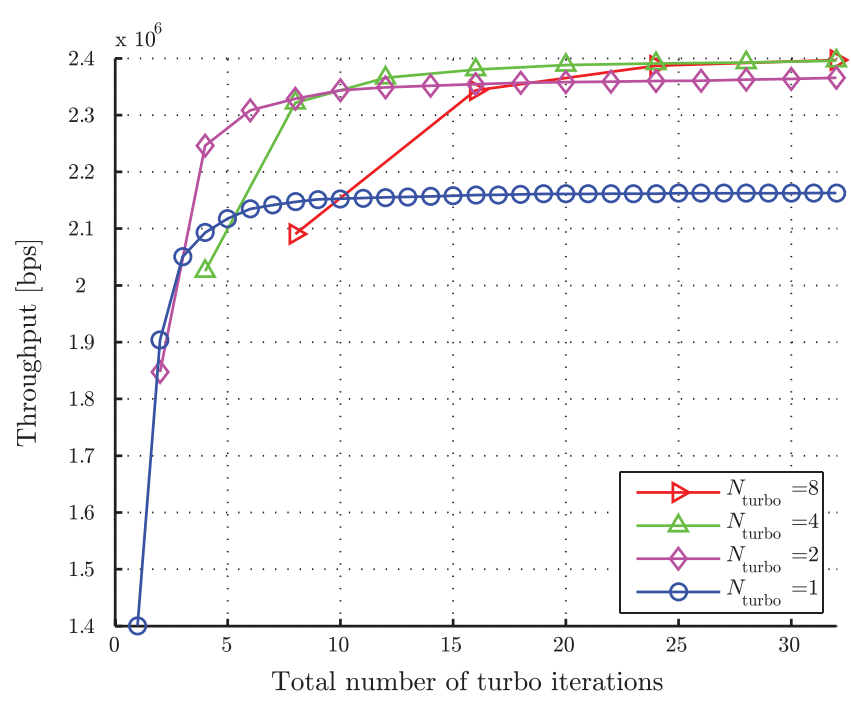

Fig. 5. Throughput versus total number of turbo iterations for $E_{c} / I_{o r}=$ $-11 \mathrm{~dB}$.

that high throughput can only be achieved with a quite large candidate list size of 128 . List sizes of 64 or smaller lead to an unstable behavior of the iterative receiver. Thus, this receiver requires a 16 times larger list size than the receiver with list updates. From (6) we obtain that the computational effort of the K-best algorithm with $K=128$ is approximately 568 flops per coded bit. With the same complexity, we could perform four list updates of the K-best-MAP with $K=8$. Comparing Fig. 4 and Fig. 6, we observe that actually two receiver iterations are sufficient with $K=8$ and list update to achieve the same performance as with $K=128$ and fixed list (the effort for the actual LLR calculation in (4), which increases with $K$, is not taken into account).

\section{Conclusions}

We proposed to use a CRC assisted K-best-MAP algorithm within an iterative receiver for MIMO-HSDPA. Throughput simulations based on the PARC proposal revealed that the K-best-MAP algorithm achieves near-optimum performance even for small list sizes $K$. We observed that the computational complexity of the K-best-MAP algorithm tends to be larger than that of one turbo decoder iteration. To achieve a prescribed performance target with minimal complexity, few outer receiver iterations with many inner turbo decoder iterations should be performed. It was also seen that without list update, much larger list sizes are required, resulting in increased overall complexity.

\section{ACKNOWLEDGMENTS}

The authors would like to thank Joachim Wehinger for his support on adapting the turbo decoder for the iterative receiver.

This work has been funded by the Christian Doppler Laboratory for Design Methodology of Signal Processing Algorithms, Kplus, Infineon Technologies, ARC Seibersdorf Research $\mathrm{GmbH}$, and the EU-FP6 project MASCOT (IST26905).

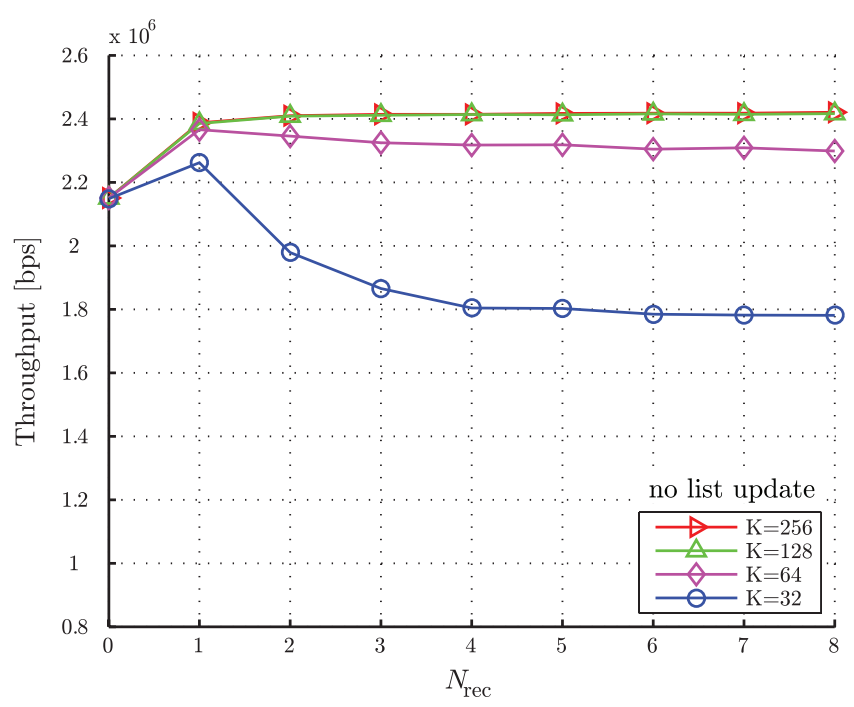

Fig. 6. Throughput versus $N_{\text {rec }}$ without list update for various $K$ and $E_{c} / I_{o r}=-11 \mathrm{~dB}$.

\section{REFERENCES}

[1] H. Huang, S. Venkatesan, A. Kogiantis, and N. Sharma, "Increasing the peak data rate of $3 \mathrm{G}$ downlink packet data systems using multiple antennas," in Proc. IEEE Vehicular Technology Conference, 2003, vol. 1, pp. $311-315$, Apr. 2003.

[2] 3GPP, "Technical specification group radio access network; MultipleInput Multiple Output in UTRA," 3GPP, Tech. Rep. 25.876 V1.7.0, Aug. 2004.

[3] B. M. Hochwald and S. ten Brink, "Achieving near-capacity on a multiple-antenna channel," IEEE Transactions on Communications, vol. 51, no. 3, pp. 389-399, Mar. 2003.

[4] H. Vikalo, B. Hassibi, and T. Kailath, "Iterative decoding for MIMO channels via modified sphere decoding," IEEE Transactions on Wireless Communications, vol. 3, no. 6, pp. 2299-2311, Nov. 2004.

[5] Z. Guo and P. Nilsson, "A low complexity soft-output MIMO decoding algorithm," in Proc. IEEE/Sarnoff Symposium on Advances in Wired and Wireless Communication, 2005, pp. 90 - 93, Apr. 2005.

[6] D. L. Ruyet, T. Bertozzi, and B. Ozbek, "Breadth first algorithms for APP detectors over MIMO channels," in Proc. IEEE International Conference on Communications, ICC 2004, vol. 2, pp. 926 - 930, June 2004.

[7] S. Bäro, J. Hagenauer, and M. Witzke, "Iterative detection of MIMO transmission using a list-sequential (LISS) detector," in Proc. IEEE International Conference on Communications, 2003, vol. 4, pp. 2653 - 2657, May 2003.

[8] Y. L. C. de Jong and T. J. Willink, "Iterative tree search detection for MIMO wireless systems," IEEE Transactions on Communications, vol. 53, no. 6, pp. 930-935, June 2005.

[9] B. Hassibi and H. Vikalo, "On the sphere-decoding algorithm I. expected complexity," IEEE Transactions on Signal Processing, vol. 53, no. 8, pp. 2806-2818, Aug. 2005.

[10] K. W. Wong, C. Y. Tsui, R. S. K. Cheng, and W. H. Mow, "A VLSI architecture of a K-best lattice decoding algorithm for MIMO channels,' in Proc. IEEE International Symposium on Circuits and Systems, 2002, vol. 3, May 2002.

[11] Z. Wang, S. Zhou, and G. B. Giannakis, "Joint coding-precoding with low-complexity turbo-decoding," IEEE Transactions on Wireless Communications, vol. 3, no. 3, pp. 832-842, May 2004.

[12] 3GPP, "Technical specification group radio access network; multiplexing and channel coding (FDD) (Tech. Spec. 25.212 V5.9.0)," June 2004.

[13] F. Kaltenberger, K. Freudenthaler, S. Paul, J. Wehinger, C. F. Mecklenbräuker, and A. Springer, "Throughput enhancement by cancelation of synchronization and pilot channel for UMTS high speed downlink packet access," in Proc. of the 6th IEEE Workshop on Signal Processing Advances in Wireless Communications (SPAWC 2005), pp. 580-584, New York, USA, June 2005.

[14] 3GPP, "Technical specification group radio access network; physical layer procedures (FDD) (Tech. Spec. 25.214 V6.2.0),” June 2004. 